\title{
Professionell und/oder gestaltungsorientiert?
}

\section{Von Anna Henkel}

Der Soziologiekongress ist einige Monate her, und wesentliche Teile der Leserschaft werden ihn selbst besucht haben. Kommentiert auch in breiter Öffentlichkeit wurde reichlich, fast will man sagen: gewohnt negativ, diesmal vor allem von der Frankfurter Allgemeine Zeitung. Was vermag man als Soziologin, als gewissermaßen berufsmäßige Beobachterin des Sozialen und sozialer Gesetzmäßigkeiten, über diese soziale Tatsache des Bamberger Soziologiekongresses noch oder überhaupt zu berichten? Wenn dem Bericht, wie hier, keine methodisch angelegte Untersuchung zugrunde liegt, die Beobachtungen also ebenso selektiv wie subjektiv bleiben, ist es aus der Perspektive einer aktiv teilnehmenden Beobachterin und Liebhaberin der soziologischen Wissenschaft: eine Reflexion auf den Kongress als Momentaufnahme der deutschsprachigen Soziologie.

Nimmt man den Soziologiekongress auf diese Weise in den Blick, muss die vorausgeschickte Frage sein, was man denn erwartete. Wer nicht zum ersten Mal (oder gerade wer zum ersten Mal) an einem Soziologiekongress teilnimmt, ist vermutlich bestrebt, eine Reihe von sonst über mindestens die ganze Republik verstreuten Bekannten zu treffen, an einem mehr oder weniger zentralen Ort versammelt. Wer mit dieser Erwartung nach Bamberg gefahren ist, wurde mit an Sicherheit grenzender Wahrscheinlichkeit nicht enttäuscht. Die absolute Anzahl der Veranstaltungen ebenso wie deren Heterogenität - von „Herausforderung der Kulturwissenschaft durch die neuen sozialen Ontologien“ bis „Aktuelle Forschungsprojekte zu Sozialstruktur und sozialer Ungleichheit" - boten geeignete Gelegenheit zur Begegnung für alle, die sich dem Fach zurechnen, was im vierstelligen Teilnehmerzahlbereich auch geschah. Die Veranstaltungsorganisation war gewohnt professionell. Sie leitete zudem offenbar eingesparte Druckkosten für einen vermutlich ohnehin zu schweren Abstract-Band in eine durchgängige Versorgung mit Obst und Kuchen um. Vermissen konnte man vielleicht den Gruppenbildungseffekt, der sonst mit dem Erkennungsmerkmal der nun fehlenden SoziologiekongressTasche einherging, aber das bleibt Geschmackssache.

Dem Social-Event-Charakter kam Bamberg also nicht weniger als andere Soziologiekongresse nach. Was aber sonst kann, darf und soll ,man“ von einem Soziologiekongress erwarten, der Tausende von wissenschaftlichen GesellschaftsbeobachterInnen zusammen bringt, der eine zumindest gewisse inner- und außerwissenschaftliche Aufmerksamkeit erregt und insofern ein Bild der deutschsprachigen Soziologie im Brennglas abgibt? Zumindest wohl zweierlei: nach innen hin eine praktische Selbstdefinition der Disziplin, nach außen hin eine Selbstdarstellung als spezifischer gesellschaftlicher Bereich. Was lernen wir derart über die Soziologie in Bamberg?

Was die Selbstdefinition angeht, so hielt die Soziologie in Bamberg ihren Anspruch, die Wissenschaft des Sozialen zu sein, thematisch, theoretisch und methodisch in umfassender und in um Aktualität bemühter Weise aufrecht. Von einer ,Schließung' konnte keine Rede sein, im Gegenteil: Gerade Themenfelder wie Digitalisierung oder Nachhaltigkeit, die nicht bereits in eigenen Sektionen oder Arbeitsgruppen abgebildet sind, wurden in den verschiedensten Veranstaltungsformaten - insbesondere den Ad-hoc-Gruppen - diskutiert. Zu vielen Themen konnte man in Bamberg einen aktuellen, über den publizierten Status hinausgehenden Forschungsstand erhören.

Digitalisierung beispielsweise ist als soziales Phänomen nicht neu und auch als Untersuchungsdimension in der Soziologie nicht neu. Veränderungen von Interaktion auf der Ebene der Kommunikationswege, veränderte Bedingungen der nun möglicherweise stärker gemein-

Soziale Welt 68 (2017), S. 109 - 112, DOI: 10.5771/0038-6073-2017-1-109 
schaftlichen Wissensproduktion, eine Neudefinition der Unterscheidung von öffentlich und privat, all das ist bekannt. Dennoch konnte man gerade in Bamberg beobachten, wie Digitalisierung als soziales Phänomen neu, empirisch dicht, mit Bezug auf über-interaktionale Strukturen und durchaus auch kritisch in den Blick gerät. Sowohl die Sektion Wissenschafts- und Technikforschung als auch die Sektion Organisationssoziologie widmeten gleich beide Sektionssitzungen der Diskussion über Digitalisierungsphänomene; zudem verschrieben sich mehrere Ad-hoc-Gruppen der Thematik.

In diesen an mithin verschiedenen Orten stattfindenden Diskurs bringt die Soziologie in Bamberg all ihre disziplinspezifischen Stärken ein: ihre theoretische Pluralität, ihre methodisch-methodologische Pluralität, ihre begrifflich-analytische Präzision und das Anliegen, diese verschiedenen Kapazitäten zum gemeinsamen Phänomenverständnis nutzbar zu machen. Allein in einer Sitzung der Wissenschafts- und Technikforschung wurden etwa zur Diskussion gestellt: eine Theorie des Crowd-Sourcing, die im Anschluss an Heideggers Wasser im Wasserkraftwerk die ,Crowd' im Crowd-Sourcing als Modus des Bestellens beobachtet (Dickel / Thiem); die Untersuchung messbarer Nutzeraktivitäten als taxonomische Kollektive, bei denen das Sehen des Publikums und das sich selbst Beobachten aufeinander bezogen sind (Muhlet / Wehner); der Tourismus 2.0 im Stile von AirBnB als hybridem Erfahrungsraum, der zwischen medialer Vermittlung und digitaler Entnetzung angesiedelt ist (Frisch / Stoltenberg).

Bilden mit Crowd-Sourcing, Nutzeraktivität und Plattform-Ökonomie hier (fraglos?) neue, technisch vermittelte Phänomene den Ausgangspunkt, so stellte sich in der gleichnamigen Adhoc-Gruppe die grundsätzliche Frage, was überhaupt neu an der Digitalisierung sei. Mittels diskurstheoretischer Ansätze wurde hier die Revolutionsrhetorik der Digitalisierung in den Blick genommen und wurden die doch sehr heterogenen Formen der Digitalisierung differenziert. Zwischen realer Veränderung und im Diskurs produzierter Sachzwanglogik erweist sich „Digitalisierung“ als mit wirtschaftlichem und politischem Druck vorangetriebener Prozess (Carstensen). Auch auf der Ebene der Organisation prägen zukunftsorientierte Visionen die „Industrie 4.0“. Die Anforderung eines ,Sensemaking“ zur Orientierung einerseits, eine neue Qualität von Kontrolle am ,smarten Arbeitsplatz“ andererseits verweist auf die nur lose Kopplung zwischen Schlagwort und organisationaler Realität (Meyer / Schaub).

Diskurs und Praxis, theorieorientierte Begriffsbildung und ethnografisch ansetzende Forschung - auch in anderen Veranstaltungen wurden sie zur Erschließung des Phänomens ,der ${ }^{\star}$ Digitalisierung eingesetzt. Kann man von Plattform-Ökonomie allgemein sprechen - oder muss man nicht unterschiedliche Formen differenzieren? Ist digital vermittelte Netz-Kommunikation tatsächlich eine Alternative zu formaler Organisation - oder basiert das Netz nicht eher auf den Strukturen und der Finanzmacht der Organisation? Ja, ist die Digitalisierung der Organisation nicht vielmehr eine Übernahme von Netz-Kommunikation durch finanz-, struktur- und machtstarke formale Organisationen?

Beobachtet man anhand einer solchen Phänomen-Erschließung (ähnlich wäre dies in Bamberg auch für andere Themen möglich, etwa den Phänomenbereich Ökologie/Nachhaltigkeit) die Soziologie auf ihre Selbstdefinition hin, so wird der ebenso hohe inhaltliche wie sachlichprofessionelle Anspruch der Disziplin an sich selbst deutlich. Jedenfalls in Diskussionsfeldern wie diesen sind die Vorträge durchgängig hochkarätig im besten Sinne des Wortes - nämlich zugleich theoretisch-methodisch reflektiert und von einer ernst gemeinten Fragestellung geleitet. Die Forschergenerationen sind gemischt - wobei weder die Vortragssituationen noch die Diskussionen einen wie auch immer gearteten Prüfungscharakter haben. Man diskutiert wertschätzend und sachlich und begegnet sich auf Augenhöhe. Der hohe eigene Anspruch wird bei den Vortragenden und Diskutierenden ebenso deutlich wie bei den Veranstaltenden und Moderatoren der verschiedenen Sitzungen. 
So lässt sich zusammenfassen: Nach innen hin, gerade in den „kleinen“ Sitzungen (den Adhoc-Gruppen, den Sektionssitzungen), geht es der Soziologie um die Sache. Man kennt sich in den wissenschaftlichen Gemeinschaften, diskutiert aber auch mit denen, die man nicht kennt; Qualität der Gedankenführung und Originalität der Beobachtung scheinen deutlich ausschlaggebend. Es herrscht fasst durchweg eine angenehme Arbeitsatmosphäre, in der man sich wechselseitig berichtet, aufeinander bezieht und das gegenwärtige Themenspektrum kennenlernt.

Die praktische Selbstdefinition der Soziologie lässt sich also kaum anders als positiv beobachten, auch wenn der Snapshot aus der Digitalisierung womöglich nicht für alle Einzelveranstaltungen gleichermaßen gelten mag. Wie aber sieht es mit der Selbstdarstellung nach außen aus - und woher kommt diese beinahe schon habituell anmutende Kritik in öffentlichen Medien?

Man kann jedenfalls nicht behaupten, die Veranstalter hätten es an Prominenz oder Öffentlichkeitswirksamkeit fehlen lassen. Mit Andreas Voßkuhle wurde der Kongress bereits durch einen Hauptvortrag eröffnet, der auch in der Bamberger interessierten Öffentlichkeit auf Resonanz stieß. Mit Nancy Fraser, Richard Alba, Thomas DiPrete, Moshe Zuckermann, Jill Rubery und Gøsta Esping-Andersen war eine thematisch plurale internationale Forscherschaft auf dem Kongress vertreten. Das Kongressthema „Geschlossene Gesellschaften“ traf dabei eine aktuelle Tendenz - und da es sich um einen Kongress, nicht um ein sachthematisch eng vordefiniertes Symposium, handelte, muss es nicht verdrießen, wenn nicht jeder Vortrag in jeder Veranstaltung vordergründig genau dazu etwas sagte.

Woher also angesichts der professionellen Kongressorganisation, der prominenten Rednerschaft, der internationalen Bezüge, des aktualitätsbezogenen Rahmenthemas und der produktiven praktischen Selbstdefinition die an eher willkürlich anmutenden Einzelaspekten festgemachte Totalkritik? Auch hier mag die Frage helfen, was man denn erwartet hat - und was vielleicht legitimerweise von der Soziologie erwartbar ist. Die Antwort scheint mir im Geltungs- und damit verbunden im Gestaltungsanspruch der Soziologie zu liegen, dessen Grenzen vielleicht gerade an einem Thema wie „Geschlossene Gesellschaften“ deutlich werden.

Die Soziologie und ihre verschiedenen Ansätze und Spielarten mögen viele Gründungsszenen haben. Eine wesentliche dieser Gründungsszenen ist die ,Soziale Frage` des 19. Jahrhunderts. Es ging dabei - auch - darum, eine Gesellschaft in tiefgreifenden und durchaus furchterregenden Umwälzungen nicht nur zu beobachten, sondern über sich selbst aufzuklären. $\mathrm{Ob}$ ein politisch-kritischer Anspruch damit aktiv verbunden wurde oder nicht - schon das Verstehen und Erklären (paradigmatisch: der Arbeitslosigkeit im Mariental) ist, gerade wenn methodisch sauber und innovativ sowie theoretisch wertfrei durchgeführt, performativ ein Gestaltungsanspruch.

An sozialen Tatsachen in diesem Sinne fehlt es auch heute nicht - und es ist auch nicht so, dass sich die Soziologie mit ihnen nicht befasste. Ein Kongressthema wie „Geschlossene Gesellschaften“ zeugt von diesbezüglicher Aufmerksamkeit; um die Grundlinien der alten ,Sozialen Frage" haben sich ganze Sektionen und Theorieansätze gebildet; eine Sektion „Soziale Ungleichheit“ und eine Sektion „Soziale Probleme und Soziale Kontrolle“ widmen sich der als Kongressthema aufgeworfenen Frage ganz speziell. Mit stärker empirischem Schwerpunkt (etwa: geflüchtete Kinder) oder mit stärker sozialstrukturellem Schwerpunkt (etwa: Wohlfahrtsstaat und Migration) oder angelegentlich auch eines scheinbar technischen Themas wie Digitalisierung - die Soziologie ist aufmerksam für Ungleichheit, für Machtverhältnisse, für Ideologie, für gesellschaftlichen Wandel und befasst sich professionell, sachlich und aufgeschlossen mit entsprechenden sachthematischen Gegenständen. 
Was fehlen mag - und dies geht in die Richtung einer gesellschaftstheoretischen Hypothese - ist die Überführung des Gestaltungsanspruchs in ein im 21. Jahrhundert anschlussfähiges Gewand. Im Werturteilsstreit wurde die Frage verhandelt, ob politisch-persönliche Wertungen in die Forschung einfließen sollen oder nicht. Doch auch eine werturteilsfreie MarienthalStudie, eine Philosophie des Geldes oder eine Definition der bürokratischen Herrschaft haben in einer weithin bürgerlich geprägten Honoratiorengesellschaft einen gewissen Wirkungsgrad, indem sie Möglichkeiten zu verstehen und zu erklären jenen nahelegen, die (ob zugerechnet oder nicht) gesellschaftlich entscheiden und handeln.

Gerade die Soziologie hat beobachtet, dass sich Gesellschaft massiv wandelt-bürokratisiert, ökonomisiert, rationalisiert, entfremdet, versachlicht, medialisiert etc. Und sie hat sich doch in dieser Entwicklung - um es salopp zu sagen - von der Ökonomie weithin den Rang ablaufen lassen. Zwar bleibt Soziologie die Wissenschaft des Sozialen, und der Ökonomie geht es ,nur ${ }^{6}$ um ökonomische Gesetzmäßigkeiten; doch als Experten für soziale Phänomene werden ganz wesentlich Ökonomen herangezogen. Zwar hat die Soziologie den theoretisch und methodisch reflektierteren, dichteren, reflexiveren und kritischeren Zugriff auf das Soziale; doch aus dem disziplinären Zuständigkeitsanspruch resultiert kaum je ein gesellschaftlich gestaltungsorientierter Anspruch.

Auch in Bamberg bleibt der Eindruck, dass nach innen hin die Soziologie zwar offen, tolerant und professionell arbeitet, nach außen hin aber vermag sie ihren, dem disziplinären Selbstbewusstsein und ihrem Professionalisierungsgrad angemessenen, Gestaltungsanspruch kaum zu vertreten. Und die Soziologinnen und Soziologen nehmen dies weithin hin, denn eine fortschreitende gesellschaftliche Rationalisierung wird in der Soziologie zwar (kritisch) beobachtet, zugleich aber eine sukzessive Zunahme quantitativ-empirischer Sozialforschung zugelassen, und das einhergehend mit einer Soziologie, die sich in heterogenen Fragestellungen, Theorieschulen und zugehörigen Zeitschriften zu verlieren droht. Damit die Soziologie in der modernen - rationalisierten, funktional differenzierten, bürokratisierten etc. - Gesellschaft des 21. Jahrhunderts die ihrem theoretisch-methodischen Reflexionsniveau angemessene Aufmerksamkeit erfährt, bedürfte es einer Transformation des soziologischen Gestaltungsanspruchs von einer ,werturteilsfreien“ Analyse zu einer zwar ,werturteilsfreien', gleichwohl aber urteilenden und empfehlungsorientierten Analyse: Dies ist keine Aufforderung, den Werturteilsstreit wieder aus der Mottenkiste zu holen. Es ist aber eine Aufforderung, mit der soziologischen Analyse nicht nur - mit Luhmann formuliert - sich als Soziologie selbst gegenüber einem von ihrer Umwelt abgeschlossenen System zu reproduzieren, sondern die Gesellschaft als ebenso irritierende wie zu irritierende Umwelt ernst zu nehmen.

Prof. Dr. Anna Henkel Leuphana Universität Lüneburg Institut für Soziologie und Kulturorganisation Professur für Kultur- und Mediensoziologie

Scharnhorststraße 1 21335 Lüneburg anna.henkel@leuphana.de 